\title{
Noon ionospheric signatures of a sudden commencement following a solar wind pressure pulse
}

\author{
A. Vontrat-Reberac ${ }^{1}$, J.-C. Cerisier ${ }^{1}$, N. Sato ${ }^{2}$, and M. Lester ${ }^{3}$ \\ ${ }^{1}$ CETP/CNRS, Paris, France \\ ${ }^{2}$ National Institute of Polar Research, Tokyo, Japan \\ ${ }^{3}$ University of Leicester, UK
}

Received: 31 October 2001 - Revised: 7 January 2002 - Accepted: 10 January 2002

\begin{abstract}
Using experimental data from the Cutlass SuperDARN HF radars and from a subset of ground magnetometers of the IMAGE Scandinavian chain, the response of the ionosphere in the noon sector to a solar wind pressure increase is studied. The emphasis is on the signature of the convection vortices and of the Hall currents that are associated with the pair of opposite parallel currents flowing along the morning and afternoon high-latitude magnetic field lines. We show that the sudden commencement is characterised by an equatorward convection, immediately followed (within less than $3 \mathrm{~min}$ ) by a strong poleward plasma motion. These results are shown to agree qualitatively with the global model of sudden commencement of Araki (1994).
\end{abstract}

Key words. Ionosphere (plasma convection; electric fields and currents) - Magnetospheric physics (solar windmagnetosphere interactions)

\section{Introduction}

Transient phenomena in the daytime ionosphere represent the low altitude response to solar wind interactions with the magnetosphere. The most important driving mechanism of ionospheric convective flows is magnetic reconnection at the magnetopause. Poleward (antisunward) pulsed ionospheric flows (also called flow channels) have long been observed by radars around noon (Provan et al., 1998). They are usually attributed to pulsed and localized reconnection at the dayside magnetopause, triggered by the $B_{Z}$ component of the interplanetary magnetic field (Pinnock et al., 1993; Lockwood et al., 1993). Solar wind dynamic pressure changes are a second source of transient phenomena. They have been shown to produce transient magnetosphere and ground signatures, called sudden commencements (sc), because they appear instantaneously (within a few minutes) over the globe. At high-latitudes, their signatures closely resemble those

Correspondence to: J.-C. Cerisier

(jean-claude.cerisier@cetp.ipsl.fr) of pulses of transient reconnection or Flux Transfer Events (FTEs) (Sibeck, 1990; Sibeck and Croley, 1991; Kivelson and Southwood, 1991; Thorolfsson et al., 2001).

A conceptual model of the ionospheric and ground responses to a sharp increase in the solar wind dynamic pressure has been proposed, based on the propagation of MHD waves through the magnetosphere (see Araki, 1994 and references therein). This model has been numerically evaluated by Osada (1992). In this model, the pressure increase gives rise to a compression of the magnetosphere, which is accompanied by an additional dawn to dusk current flowing in the magnetopause. This perturbation launches a fast mode compressional wave that propagates radially towards the ionosphere. Since the plasma in the magnetosphere is inhomogeneous, regions of sharp density gradients allow for the fast mode to couple to an Alfvén mode which propagates to the ionosphere along the magnetic field lines.

Due to a shorter propagation delay of the Alfvén wave, compared to the compressional wave (Kikuchi, 1986), the first response of the ionosphere (called the preliminary impulse, or PI) is due to the field-aligned currents (FACs) associated with the Alfvén wave (Wilken et al., 1982). In the Northern Hemisphere, the FACs flow into the ionosphere on the dusk side and out of the ionosphere on the dawn side. This creates a twin vortex current system in the ionosphere, with a clockwise Hall current vortex on the afternoon side and an anti-clockwise vortex on the morning side (Fig. 1).

As the compressional wave propagates towards the Earth, the magnetic field in the magnetosphere will be increased. This effect has been measured at the dayside geosynchronous orbit (Erlandson et al., 1991; Korotova et al., 1999). Once the fast wave hits the ionosphere, the H-component of the ground magnetic field starts to increase. After the compressional front has passed the Earth, the magnetospheric convection readjusts to the new compressed state. The FACs associated with this adjustment (called the main impulse, or MI) flow out of the ionosphere on the dusk side and into the ionosphere on the dawn side. This creates an ionospheric current vortex system that has an inverse sense of rotation 


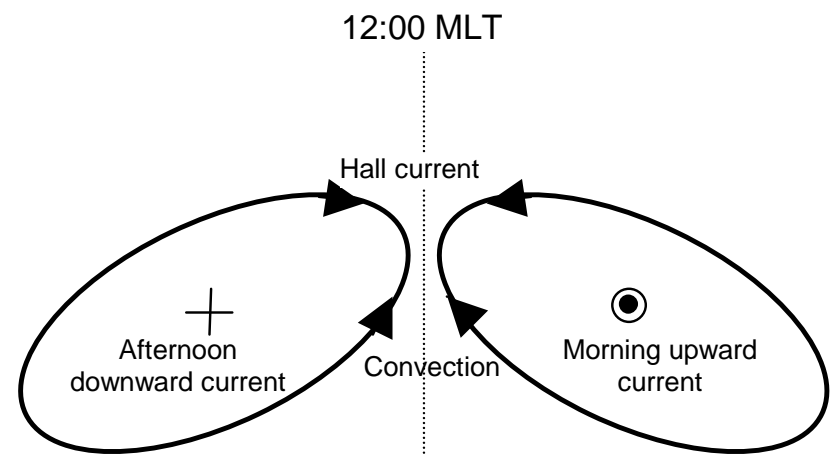

Fig. 1. Schematics of the convection-current system in the Northern Hemisphere ionosphere during the preliminary impulse. The system is reversed during the main impulse.

compared with the PI.

The ground magnetometer responses to these combined changes are mainly detected in the H-component. In the afternoon sector at mid- and auroral latitudes, the preliminary response to a sudden pressure increase is typically a short negative pulse followed by a broad positive one. In the morning sector, the signatures are reversed: a short positive pulse is followed by a broad negative pulse. Furthermore, at latitudes higher than the FACs, the sign of the magnetic pulses is reversed compared with the mid-latitude and auroral regions (Araki, 1994). This type of ionospheric response to a sudden pressure increase is referred to as the SI model.

In a 3D MHD simulation of the response of the magnetosphere to a solar wind pressure pulse, Slinker et al. (1999) have confirmed that travelling convection vortices develop on both sides of the magnetosphere with the senses described by Araki. Thorolfsson et al. (2001) have shown that convection and magnetometer data measured during a series of pressure pulses agree qualitatively with Araki's model. In an attempt to determine experimentally the latitude of the field-aligned currents, Lam and Rodger (2001) have shown that the latitude of the parallel currents varies with MLT, contrary to Araki's model, which places the parallel currents at a constant latitude, arbitrarily set to $75^{\circ}$ by Osada (1992). Using a wide set of magnetometers, Engebretson et al. (1999) have shown that the initial velocity of propagation of the PI away from noon is very large, of the order of $150 \mathrm{~km} / \mathrm{s}$.

In the noon sector, the morning and afternoon Hall current vortices merge into a northward current during the PI and a southward current during the MI, as confirmed by the simulations of Slinker et al. (1999). The main ground magnetic signature at high-latitudes is then expected to occur on the $\mathrm{D}$ (or Y) component, with a negative pulse during the PI and a positive pulse during the MI.

In this paper, the response of the ionospheric convection in the noon sector to a solar wind pressure increase occurring on 1 February 1998 is studied. The experimental data are from the Cutlass SuperDARN (Dual Auroral Radar Network) HF radars and from a set of ground magnetometers. These data are then discussed in the framework of Araki's model. The

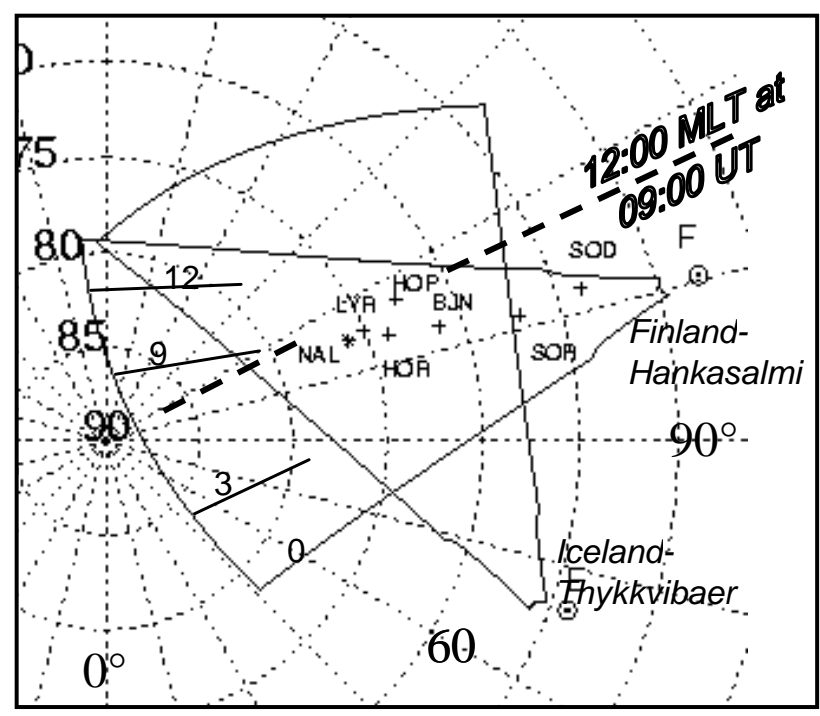

Fig. 2. Geometry of the fields of view of the CUTLASS radars at Thykkvibaer (E) and Hankasalmi (F) in AACGM coordinates. The directions of the beams of the Hankasalmi radar displayed in Fig. 5 are indicated. The position of the magnetometer stations is also indicated. The thick dashed line shows the 12:00 MLT meridian at 09:00 UT.

SuperDARN data have been already described in a paper by Ogawa et al. (2001) devoted to the study of the formation of polar cap ionisation patches (their event 2). We will use some of their results in the discussion.

\section{Instruments}

On 1 February 1998, the CUTLASS pair of the SuperDARN HF radars (Greenwald et al., 1995) was operated in a special mode consisting of a 15-beam scan. The CUTLASS radars at Thykkvibaer in Iceland and at Hankasalmi in Finland share a common field of view both over Scandinavia and north of Scandinavia. Figure 2 shows the geometry of the fields of view of the radars in Altitude Adjusted Corrected Geomagnetic (AACGM) coordinates. Data from the northern part of the International Monitor for Auroral Geomagnetic Effects (IMAGE) north-south magnetometer chain are used. At the time of the event described here (around 09:00 UT), the IMAGE magnetometers are situated in the 11:30-12:00 MLT sector, as shown in Fig. 2. The low-latitude magnetic data are from the Hermanus magnetometer, situated at 10:12 MLT at 09:00 UT.

\section{Observations}

\subsection{Solar wind conditions}

Figure 3 shows interplanetary magnetic field (IMF) and solar wind dynamical pressure data from 07:00 to 09:00 UT, obtained by the Wind satellite. This time interval is charac- 


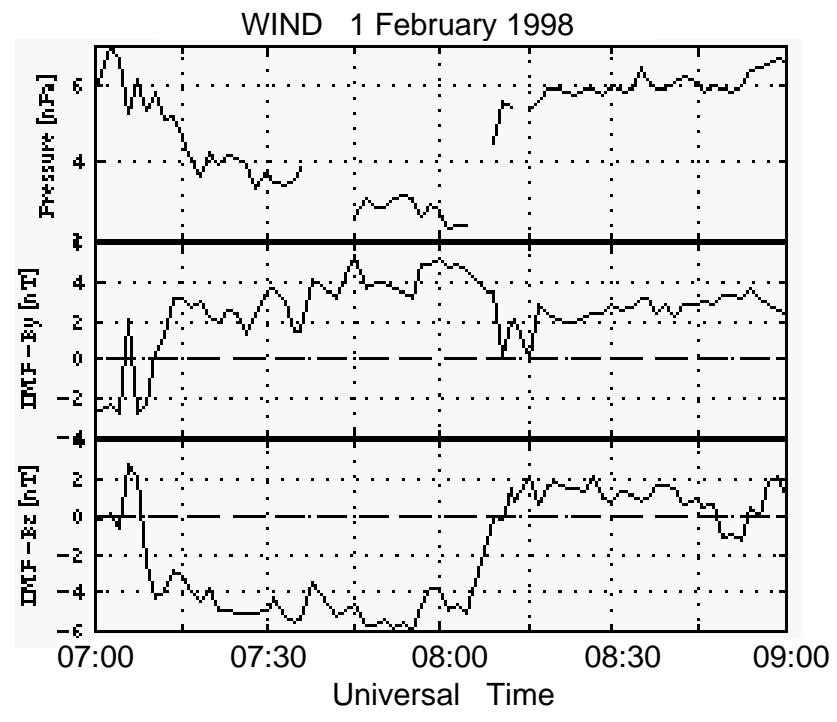

Fig. 3. The solar wind dynamic pressure and IMF components $B_{Y}$ and $B_{Z}$ (in GSM coordinates) measured by Wind during the 07:00 to 09:00 UT period.

terised by a southward IMF starting at around 07:09 UT ( $B_{Z}$ $\approx-5 \mathrm{nT}$ ) and lasting about one hour, while the solar wind dynamical pressure slowly decreases to about $2 \mathrm{nPa}$. The $B_{Y}$ component is positive with a mean value of $3 \mathrm{nT}$.

We are focussing on a particular event in the solar wind: the strong increase in the solar wind dynamic pressure $(+130 \%)$, which begins at $\sim 08: 04 \mathrm{UT}$, at the end of the southward IMF period. The duration of the pressure increase is $\sim 7 \mathrm{~min}$. At that time, the Wind spacecraft was on the dusk side of the magnetosphere $\left(X_{g s m}=235.7 R_{E}, Y_{g s m}=\right.$ $\left.8.5 R_{E}, Z_{g s m}=-26.6 R_{E}\right)$. With a solar wind velocity of $470 \mathrm{~km} . \mathrm{s}^{-1}$, the delay between the Wind spacecraft and the magnetopause can be crudely estimated to be $\sim 54 \mathrm{~min}$. Therefore, we expect to observe a response to this pressure pulse on the magnetometers around 09:00 UT.

Similar IMF and pressure signatures are also observed with the appropriate delays by IMP-8 and Interball-tail, which were separated by $\sim 30 R_{E}$ transverse to the Sun-Earth direction, thus showing the large-scale extent of the pressure pulse. Geotail, on the morning side of the magnetosphere, at $\left(X_{g s e}=8 R_{E}, Y_{g s e}=-4.7 R_{E}, Z_{g s e}=1 R_{E}\right)$, enters the magnetosheath between 08:58 and 09:00 UT, thus dating clearly the compression of the magnetosphere.

\subsection{Ground magnetometer data}

Figures $4 \mathrm{a}$ and $4 \mathrm{~b}$ show the components in the direction of magnetic north and magnetic east (referred to as $X_{m}$ and $Y_{m}$, respectively) of several high- and middle-latitude magnetic stations from the IMAGE chain (NAL, LYR, HOR, HOP, BJN, SOR and SOD) between 08:20 and 09:40 UT on 1 February 1998. This particular projection of the horizontal magnetic component is chosen to facilitate the comparison with radar data. Figures $4 \mathrm{a}$ and $4 \mathrm{~b}$ also show the usual $X$ and $Y$ components at the low-latitude dayside station Hermanus (HER, $-33.7^{\circ}$ geomagnetic latitude). The stations are ordered in decreasing latitudes, from top to bottom, with a different scale for the components measured at HER.

In response to the pressure increase measured by Wind at around 08:04 UT, the IMAGE and HER stations show a typical sc response. A sudden increase in the $X_{m}$ component at mid-latitude (SOR, SOD), and of the $X$ component at lowlatitude (HER) is observed at 08:58 UT. The $X_{m}$ component also shows a double pulse structure at the northern most stations (HOR, LYR, NAL), with a negative deflection during the MI. At the lower latitude IMAGE stations, no clear signature is present during the $\mathrm{PI}$, and the $X_{m}$ component shows only a positive pulse during the MI. This change in the sign of the MI signature suggests that the MI parallel current is centred between HOR and HOP, around $73^{\circ}$ magnetic latitude (Fig. 2).

At high-latitudes, deflections of the $Y_{m}$ component are dominant, with the maximum amplitude of the $Y_{m}$ component being observed at HOR. This component is mainly affected by a north-south Hall current. All stations show a negative $Y_{m}$ deviation centred on 09:01 UT, followed by a positive $Y_{m}$ deviation centred on 09:05 UT. Attributing these magnetic pulses respectively to the PI and to the MI pulses agrees with Araki's model of a poleward current during the $\mathrm{PI}$ and an equatorward current during the MI.

\subsection{HF radar data}

Figure 5 shows the radial velocities measured by the Hankasalmi radar for 3 selected beams (beams 3-9-12) between 08:20 and 09:40 UT. Beams 3 and 9 have a higher time resolution than beam 12. The field of view of the radar is centred on the cusp region (11:00 MLT at 09:00 UT, see Fig. 2), with mainly negative (poleward) velocities. During the southward IMF period (before 09:00 UT), clear poleward moving flow bursts are observed on beam 3 (for instance, between 08:36 and 08:40 UT). From the analysis of the occurrence of these flow bursts, which are normally regarded as an ionospheric signature of transient reconnection, the cusp can be situated between 09:00 and 12:00 MLT. At 09:00 UT, positive (equatorward) velocities reaching $700 \mathrm{~m} / \mathrm{s}$ are observed on beams 9 and 12, in the eastern part of the field of view. This corresponds to the time of the PI, as seen by the magnetometers. Large negative velocities, up to $1200 \mathrm{~m} / \mathrm{s}$, are observed immediately after, centred on 09:05 UT, on beams 3 and 9, in the central and western parts of the field of view. They can be associated with the MI magnetometer signature.

In order to describe in more detail the ionospheric flow during the sc event, Fig. 6 shows the radial velocity maps from the Hankasalmi radar at three selected times: before, during and after the event. Figure 6a shows the convection before the sc. The change from slightly positive velocities in the eastern beams to more intense negative velocities on the western beams results mainly from a rotation of the flow from essentially perpendicular to the beam direction to parallel to the beam direction. This is consistent with a round 
(a) 1 February 1998

IMAGE $X m$ and Hermanus $X$ components (nT)

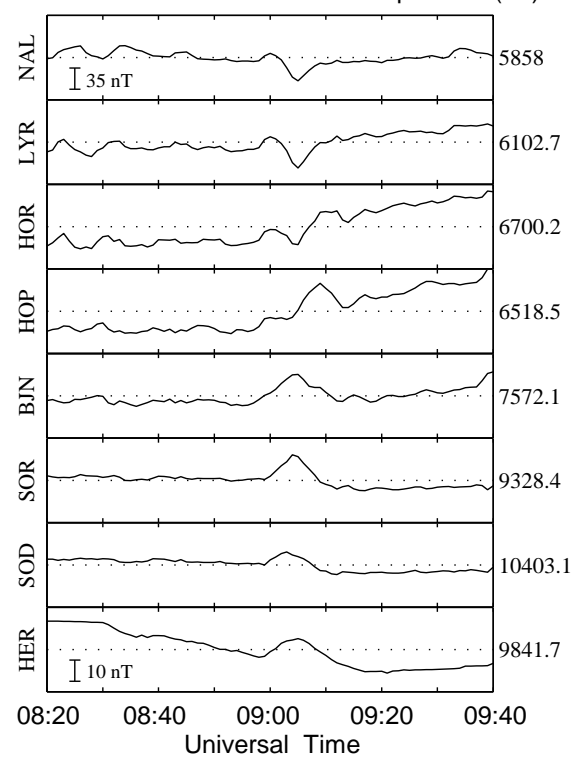

(b) 1 February 1998

IMAGE $Y m$ and Hermanus $Y$ components (nT)

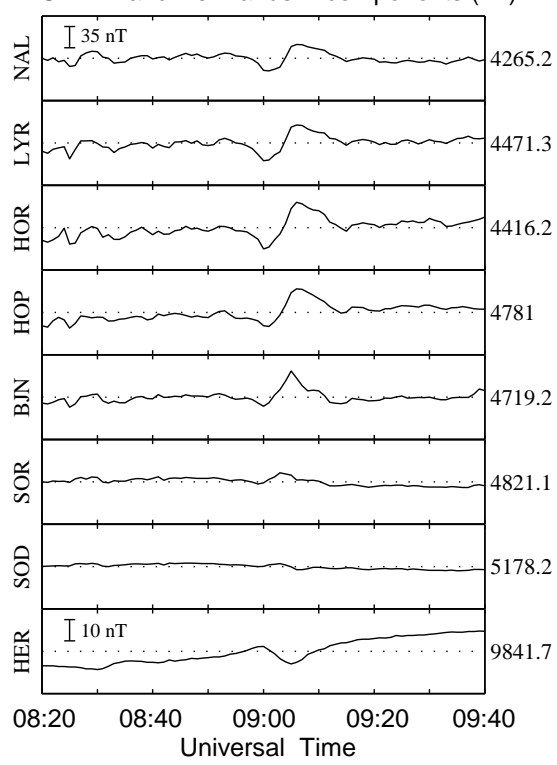

Fig. 4. Magnetometer data from the IMAGE north-south chain and from Hermanus (South Africa).

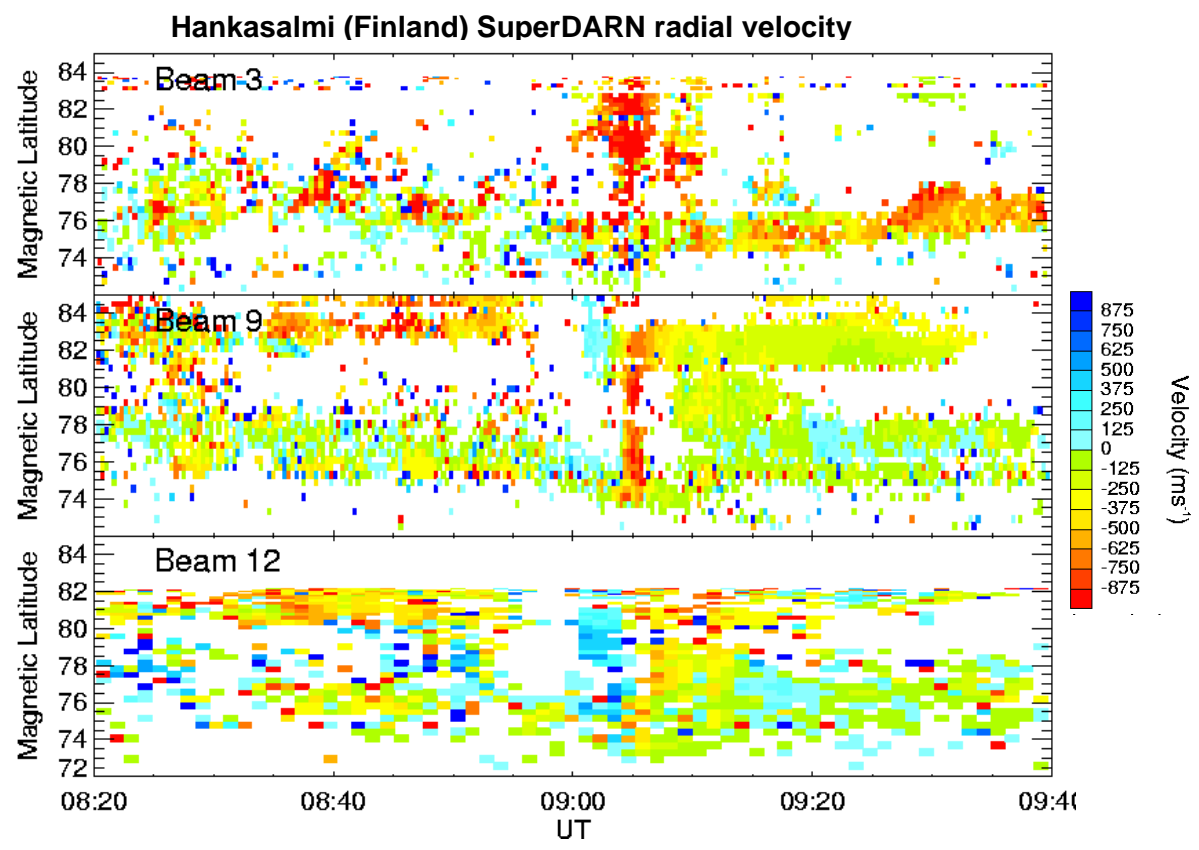

Fig. 5. Radial velocities in 3 beam directions of the Hankasalmi radar.

evening cell driving a westward flow into the polar cap, characteristic of a positive IMF $B_{y}$.

As previously discussed with Fig. 5, the 09:01 UT map (Fig. 6b) during the PI shows positive velocities in the eastern part of the field of view (beams 9 to 14) in the 14:00 MLT sector. Simultaneously, the Thykkvibaer radar (not shown, see Plate 3 of Ogawa et al., 2001) observes strong westward velocities. At the same time, the northern most IMAGE magnetometers observe positive $X_{m}$ and negative $Y_{m}$ deviations, in qualitative agreement with southward and westward convection.
At 09:05 UT (Fig. 6c), the convection is considerably changed, with strong negative (poleward) velocities observed on most beams of the Hankasalmi radar. Again, these poleward velocities are in qualitative agreement with the positive $Y_{m}$ observed at all IMAGE stations. In addition, the Thykkvibaer radar shows negative (eastward) velocities at far ranges in the northern most beams, in agreement with the negative $X_{m}$ component at the northern most IMAGE magnetometers. At the lowest latitude IMAGE stations (HOP, BJN, SOD), the $X_{m}$ component is positive, in agreement with a westward component of the convection. 


\section{Hankasalmi (Finland) SuperDARN radial velocity}
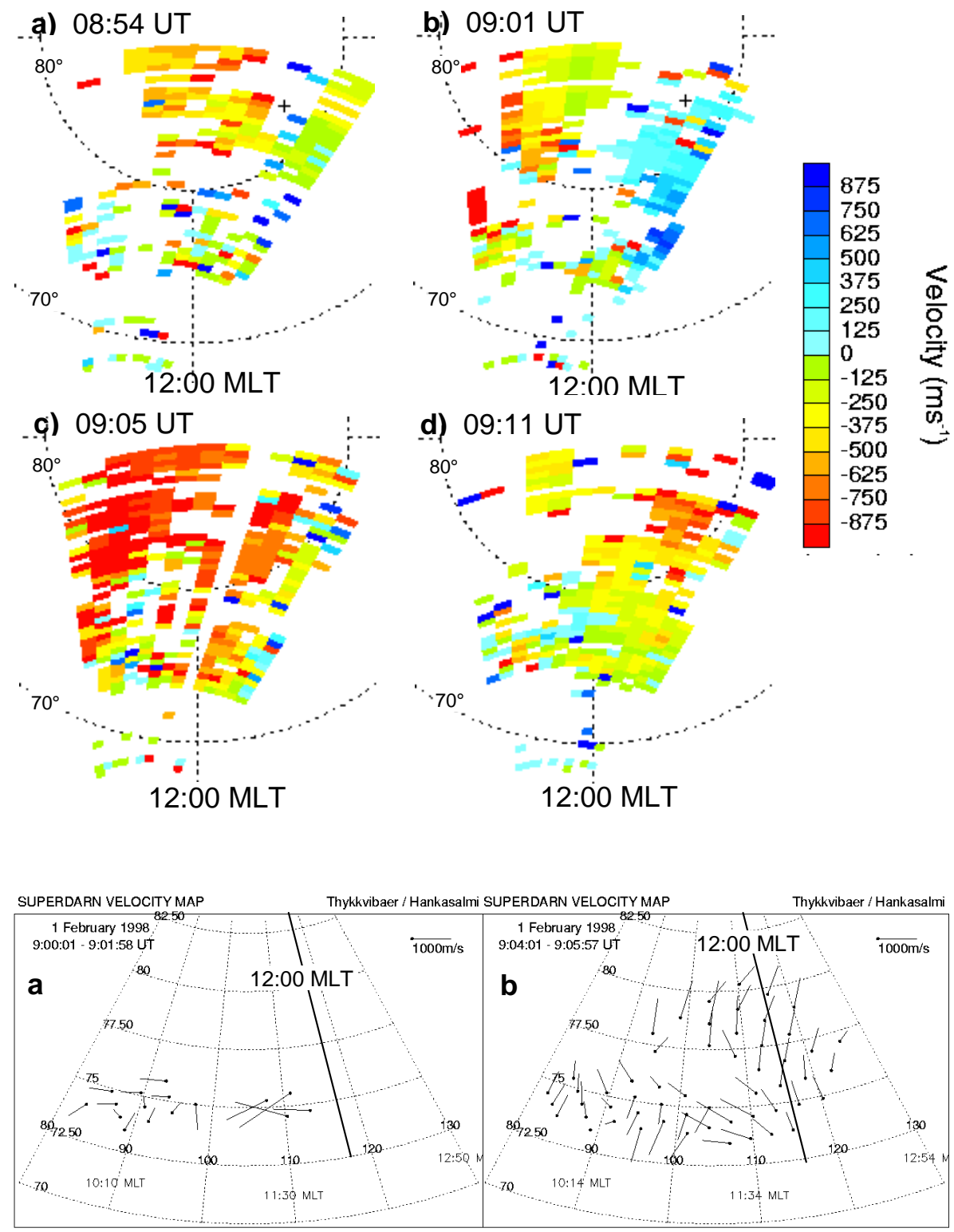

Fig. 6. Selection of radial velocity maps from the Finland-Hankasalmi radar; (a) 08:54 UT before the sc; (b) 09:01 UT during the PI; (c) 09:05 UT during the MI; (d) 09:11 UT after the sc. Positive velocities are towards the radar.

Fig. 7. Vector velocity map deduced from the Cutlass radar pair. (a) During the preliminary impulse; (b) during the main impulse. The thick line shows the 12:00 MLT meridian at 09:00 UT.
The last map at 09:11 UT, after the sc (Fig. 6d), shows a low velocity westward convection in the $73-76^{\circ}$ latitude range. These low velocities indicate that the convection did not return to its initial state prior to the sc, but rather turns to a state more in agreement with what is usually expected for positive IMF $B_{Z}$. Large, negative velocities remain only in the far polar cap. Figure 7 shows vector velocity maps deduced from simultaneous measurements by the two Cutlass radars during the periods identified as the PI (09:0009:02 UT) and the MI (09:04-09:06 UT). These maps help to understand the relation between the IMAGE and the SuperDARN data. Figure 7a shows that, during the PI, a westward flow (with velocities in excess of $1500 \mathrm{~m} / \mathrm{s}$ ) exists around $75^{\circ}$ (and around 11:30MLT), which is responsible for the positive $X_{m}$ deflection in the northern IMAGE magnetometers.
The absence of vectors associated with the southward flow observed in the eastern part of the field of view of the Hankasalmi radar is due to the absence of scatter at the large ranges of the Iceland radar. During the MI (Fig. 7b), the northward flow is clearly observed, with velocities rotating from slightly westward below $75^{\circ}$ to slightly eastward above $76^{\circ}$ magnetic latitude.

\section{Discussion and conclusion}

Transient flows around noon are usually associated either with IMF or with pressure pulse effects. We will first show that the transients observed between 09:00 and 09:10 UT cannot be IMF effects. 
The 09:05 UT poleward flow does not present any of the characteristics of the transient poleward flows that are observed in the period before 09:00 UT and usually present in the cusp under negative IMF $B_{Z}$ and have been attributed to flux transfer events: these are spatially localised with a maximum latitudinal extent of a few hundred km (Provan et al., 1998; Thorolfsson et al., 2000).

As it often happens, the solar wind pressure increase does not occur alone, but is associated with a change in the orientation of the IMF. In the present case, the GSM $B_{Z}$ component turns from negative to slightly positive. It is tempting to attribute the observed transients to this IMF change. In the framework of this interpretation, the equatorward convection observed at 09:01 UT could then be attributed to lobe reconnection driving sunward convection in the noon polar cap. This interpretation can, however, be ruled out for three reasons: (1) the lobe cell is usually observed for strongly northward IMF while, in the present case, the IMF is mainly duskward; (2) the transient nature of the equatorward flow does not agree with the stable positive IMF prevailing after 09:00 UT; (3) after a positive $B_{Z}$ turning, a slow decay of convection velocities usually takes place, as observed between 09:08 and 09:20 UT. But this disagrees with the strong poleward flow observed at 09:05 UT, after the equatorward flow.

We now discuss our observations in the framework of Araki's model (1994). The change in the sign of the $X_{m}$ component, from negative for NAL and LYR to positive for BJN and SOR, suggests that the MI parallel current was located at a magnetic latitude of $73^{\circ}$ AACGM, against $75^{\circ}$ which was arbitrarily chosen in Araki's model. Previous experimental studies tend to indicate lower latitudes, $70^{\circ}$ in the work of Thorolfsson et al. (2001), and below $72^{\circ}$ in the work of Lam and Rodger (2001). The latitude of the parallel currents can also be inferred from the convection pattern. During the MI (Fig. 7b), the convection velocity has turned eastward above $75^{\circ}$ magnetic latitude, whereas it was strongly westward before the sc. This indicates that the MI parallel current on the evening cell is certainly situated below $75^{\circ}$. Thorolfsson et al. (2001) have observed that the latitude of the MI current was $5^{\circ}$ below the convection reversal boundary (CRB). The present results appear different: the CRB is observed at $\sim 74^{\circ}$ both at Hankasalmi and at earlier MLTs by the Stokkseyri SuperDarn radar (not shown). This value is just one degree above our evaluation of the MI parallel current latitude. A DMSP-F11 pass at 08:30 UT in the early afternoon sector shows a sharp decrease in the electron fluxes and mean energy at $73.7^{\circ}$, together with a disappearance of the highenergy ions $(>10 \mathrm{keV})$ at $74.2^{\circ}$. This suggests that the MI current is related to the density gradient associated with the precipitation boundary (plasma sheet outer boundary).

The PI signature is less clear. No clear change in the sign of the $X_{m}$ magnetic component is observed: the positive $X_{m}$ signature observed at the northern most IMAGE stations has no negative counterpart at lower latitudes. This absence of a clear localisation of the PI current has also been remarked by Lam and Rodger.
The central MLT of both flows can be inferred from the velocity maps at 09:01 and 09:05 UT on Figures 6-b and 6-c. In fact, the PI is centred at 12:00 MLT and the MI at 11:00 MLT. This difference can be explained by the different origins of the vortices during PI and MI. The MLT location of the PI flow is related to the position on the magnetosphere of the initial impact of the pressure pulse, and thus to the orientation of the pressure front in the solar wind, as suggested by Takeuchi et al. (2000). On the other hand, the MI, which is due to the readjustment of the convection to the new solar wind pressure, is expected to be more symmetric with respect to the noon-midnight plane.

In conclusion, we have studied the noon high-latitude ionospheric signatures of a positive pressure pulse in the solar wind. We have shown that the associated latitudinal Hall currents and convective flows lead to consistent magnetometer and radar signatures. These signatures also agree with the global model of Araki (1994).

Acknowledgements. The CUTLASS SuperDARN radars are supported by the Particle Physics and Astronomy Research Council (PPARC) in the UK, the Swedish Institute of Space Physics, Uppsala, and the Finnish Meteorological Institute, Helsinki. The research at CETP was supported by Institut National des Sciences de l'Univers (INSU) in France. We thank R. Lepping and K. Ogilvie for providing respectively the Wind solar wind IMF and plasma data through the Coordinated Data Analysis Web (CDAWeb). We thank D. Hardy, F. Rich and P. Newell for the use of DMSP particle data. The IMAGE magnetometer data are provided through the Finnish Meteorological Institute, and the Hermanus magnetometer data are provided through the World Data Center for Geomagnetism in Kyoto. We thank A. Thorolfsson for useful comments.

The Editor in Chief thanks T. Takeuchi and another referee for their help in evaluating this paper.

\section{References}

Araki, T.: A physical model of the geomagnetic sudden commencement, in Solar wind sources of Magnetospheric Ultra-Low Frequency Waves, Geophys. Monog. Ser., vol 81, edited by M. J. Engebretson, K. Takahashi, and M. Scholer, 183-200, AGU, Washington D. C., 1994.

Engebretson, M. J., Murr, D. L., Hughes, W. J., Lühr, H., Moretto, T., Posch, J. L., Weatherwax, A. T., Rosenberg, T. J., McLennan, C. J., Lanzerotti, L. J., Marcucci, F., Dennis, S., Burns, G., Bitterly, J., and Bitterly, M.: A multipoint determination of the propagation velocity of a sudden commencement across the polar ionosphere, J. Geophys. Res., 104, 22 433-22 451, 1999.

Erlandson, R. E., Sibeck, D. G., Lopez, R. E., Zanetti, L. J., and Potemra, T. A.: Observations of solar wind pressure initiated fast mode waves at geostationnary orbit and in the polar cap, J. Atmos. Terr. Phys., 53, 231-239, 1991.

Greenwald, R. A., Baker, K. B., Dudeney, J. R., Pinnock, M., Jones, T. B., Thomas, E. C., Villain, J.-P., Cerisier, J.-C., Senior, C., Hanuise, C., Hunsucker, R. D., Sofko, C., Koehler, J., Nielsen, E., Pellinen, R., Walker, A. D. M., Sato, N., and Yamagishi, H.: DARN/SuperDARN: A global view of high latitude convection, Space Sci. Rev., 71, 761-796, 1995. 
Kikuchi, T.: Evidence of transmission of polar electric fields to the low-latitude at times of geomagnetic sudden commencements, J. Geophys. Res., 91, 3101-3105, 1986.

Kivelson, M. G. and Southwood, D. J.: Ionospheric travelling vortex generation by solar wind buffeting of the magnetosphere, J. Geophys. Res., 96, 1661-1667, 1991.

Korotova, G. I., Sibeck, D. G., Moretto, T., and Reeves, D. G.: Tracking transient events through geosynchroneous orbit, J. Geophys. Res., 104, 10 265-10 273, 1999.

Lam, M. M. and Rodger, A. S.: A case study test of Araki's physical model of geomagnetic sudden commencement, J. Geophys. Res., 106, 13 135-13 144, 2001.

Lockwood, M., Moen, J., Cowley, S. W. H., Farmer, A. D., Lovhaug, U. P., Lühr, H., and Davda, V. N.: Variability of dayside convection and motions of the cusp/cleft aurora, Geophys. Res. Lett., 20, 1011-1014, 1993.

Ogawa, T., Buchert, S. C., Nishitani, N., Sato, N., and Lester, M.: Plasma density suppression process around the cusp revealed by simultaneous CUTLASS and EISCAT Svalbard observations, J. Geophys. Res., 106, 5551-5564, 2001.

Osada, S.: Numerical calculation of the geomagnetic sudden commencement, M. S. Thesis, Faculty of Sciences, Kyoto Univ., Kyoto, March 1992.

Pinnock, M., Rodger, A. S., Dudeney, J. R., Baker, K. B., Newell, P. T., Greenwald, R. A., and Greenspan, M. E.: Observations of an enhanced convection channel in the cusp ionosphere, J. Geophys. Res., 98, 3767-3776, 1993.

Provan, G., Yeoman, T. K., and Milan, S. E.: Cutlass Finland radar observations of the ionospheric signatures of flux transfer events and the resulting plasma flows, Ann. Geophysicae, 16, 14111422, 1998.

Sibeck, D. G.: A model for the transient magnetospheric response to sudden solar wind pressure variations, J. Geophys. Res., 95, 3755-3771, 1990.

Sibeck, D. G. and Croley Jr, D. J.: Solar wind dynamic pressure variations and possible ground signature of flux transfer events, J. Geophys. Res., 96, 1669-1683, 1991.

Slinker, S. P., Fedder, J. A., Hughes, W. J., and Lyons, J. G.: Response of the ionosphere to a density pulse in the solar wind: simulation of travelling convection vortices, Geophys. Res. Lett., 26, 3549-3552, 1999.

Takeuchi, T., Araki, T., Lühr, H., Rasmussen, O., Waterman, J., Milling, D. K., Mann, I. R., Yumoto, K., Shiokawa, K., and Nagai, T.: Geomagnetic negative sudden impulse due to a magnetic cloud observed on 13 May 1995, J. Geophys. Res., 105, 18 835$18846,2000$.

Thorolfsson, A., Cerisier, J.-C., Lockwood, M., Sandholt, P. E., Senior, C., and Lester, M.: Simultaneous optical and radar signatures of poleward moving auroral forms, Ann. Geophysicae, 18, 1054-1066, 2000.

Thorolfsson, A., Cerisier, J.-C., and Pinnock, M.: Flow transients in the post-noon ionosphere: the role of solar wind dynamic pressure, J. Geophys. Res., 106, 1887-1901, 2001.

Wilken, B., Goertz, C. K., Baker, D. N., Higbie, P. R., and Fritz, T. A.: The SSC on 29 July 1977, and its propagation within the magnetosphere, J. Geophys. Res., 87, 5901-5910, 1982. 